\title{
Image-guided resection of high-grade glioma: patient selection factors and outcome
}

\author{
N. Scott Litofsky, M.D., Andrew M. Bauer, B.S., RaChel S. KaSPer, M.P.H., \\ Cynthia M. Sullivan, M.S., OMar H. Dabbous, M.D., M.P.H., \\ and The Glioma Outcomes Project Investigators
}

\author{
Division of Neurosurgery, University of Missouri-Columbia School of Medicine, Columbia, Missouri; \\ and The Center for Outcomes Research, University of Massachusetts Medical School, \\ Worcester, Massachusetts
}

\begin{abstract}
Object. In patients with glioma, image-guided surgery helps to define the radiographic limits of the tumor to maximize safety and the extent of resection while minimizing damage to eloquent brain tissue. The authors hypothesize that image-guided resection (IGR) techniques are associated with improved outcomes in patients with malignant glioma.

Methods. Data recorded in 486 patients enrolled in the Glioma Outcomes Project were analyzed in this study. Demographic data and outcomes in patients who underwent IGR were compared with those in patients who underwent resection without IGR. Univariate analysis performed with chi-square testing was used to compare patient presentation, tumor characteristics, and death rates. Multivariate logistic regression was used to predict various outcome parameters.

Patients who underwent IGR were younger and had smaller, lower-grade tumors than those in whom IGR was not performed. They were more likely to present with seizure and normal consciousness. Unexpectedly, gross-total resection was performed in significantly fewer patients with IGR than in individuals without IGR. Patients with IGR were more likely to be discharged home with the ability to live independently, and they had a shorter duration of hospital stay than patients without IGR. Survival was significantly longer in patients who underwent IGR, but multivariate analysis showed that glioblastoma multiforme (GBM) and age accounted for these observations.

Conclusions. Selection bias occurs regarding patients who receive IGR; these biases include younger age, presentation with seizure and normal level of consciousness, tumor diameter less than $4 \mathrm{~cm}$, and non-GBM on histopathological studies. Outcome appears to be improved in patients who undergo IGRs of high-grade gliomas. It is unclear if these improved outcomes are due to the selection of a more favorable patient population or to the IGR techniques themselves. It is likely that the full potential of image guidance in glioma surgery will not be realized until it is applied to a wider range of patients.
\end{abstract}

\section{KEY WORDS • neuronavigation • glioma • resection • neurooncology}

\section{$\mathrm{T}$} HE use of newer imaging modalities is constantly shaping the field of neurooncology. Older, static imaging systems are being augmented by newer systems with real-time and functional capabilities. This innovation in imaging technology is allowing surgeons to plan and execute more precise tumor resections and to achieve GTR in tumors for which such resections would have previously been more difficult.

As detailed in Haberland, et al., ${ }^{12}$ since the first stereotactic frame for intracranial navigation was developed by Zernow in 1889 , the field has evolved dramatically. The digital technology explosion has led to rapid advances in

Abbreviations used in this paper: GBM = glioblastoma multiforme; $\mathrm{GO}=$ Glioma Outcomes; GTR = gross-total resection; IGR = image-guided resection; KPS = Karnofsky Performance Scale; $\mathrm{MR}=$ magnetic resonance. neuronavigation. Frameless stereotactic systems have been in use in neurosurgery for a number of years. These systems are based on the traditional parameters of stereotaxy, in which the brain is separated into three planes (coronal, axial, and sagittal). The new systems then allow for threedimensional navigation within a defined space by integrating surface landmarks with merged data from radiographic images. Any point within the system can then be pinpointed using a probe and a system of detectors that reference the probe to the radiographs and project two-and threedimensional images. ${ }^{11}$

These systems can allow precision to within millimeters: ${ }^{10}$ however, they have some drawbacks. The visualization of the anatomy is confined to the state it was in when the radiographic images were obtained; most neuronavigation systems cannot yet compensate for changes in this anatomy. Surgeons encounter some discord between coordinates stored in the system and what is actually seen in the 
surgical field after some of the tumor or cerebrospinal fluid has been removed, allowing for relaxation of previously compressed tissue and shifting of the brain. Other confounding factors are cerebral edema and bleeding. Even so, these systems still pay dividends by allowing the surgeon to plan the procedure in virtual space and to have some idea of the anatomy that will be encountered.

Other technologies, such as intraoperative MR imaging and ultrasonography, also play a role in neurosurgical operating rooms. Intraoperative MR imaging has the advantage of allowing repeated acquisition of images in the patient while the craniotomy is open, enabling resection of any remaining tumor. The MR imaging modality can be used to update the information in the neuronavigational systems, ${ }^{4,10,32,33}$ thereby minimizing the confounding factor of brain shift. ${ }^{29,31}$ As higher-powered MR imaging units are adapted to the operating room, it will also be possible to use such features as functional, diffusion-weighted, and spectroscopic images, and to integrate these with neuronavigator systems.

Another technology that is proving useful in brain tumor resection is ultrasonography. Due to the difference in impedance between normal brain and tumor, it is possible to identify remnants of tumor after a resection. Ultrasonography has the advantages of lower cost, decreased operating time, and ease of use compared with intraoperative MR imaging. With newer neuronavigational systems it is possible to merge ultrasonographic data with MR imaging or computerized tomography scans, thereby augmenting the surgeon's view of the tumor. Studies have shown that ultrasonography-based systems can provide accuracy comparable to that of traditional MR imaging-based systems. ${ }^{26}$

Although these advances in technology have been amazing steps in the advancement of the field, their utility in all tumor resections has yet to be adequately defined. The role of surgery in the treatment of malignant glioma is somewhat controversial. Malignant glioma has perhaps the worst prognosis of any tumor, with the median survival duration well below 18 months, despite all advances in technology and treatment. ${ }^{28}$ There is an accruing body of evidence (although this evidence is "not of high quality") ${ }^{18}$ showing a survival advantage for extensive resection at the time of diagnosis in patients with low-grade gliomas. ${ }^{19}$ The correlation of the extent of resection with survival in patients with high-grade gliomas is even less well defined. A number of studies detail a favorable effect of early, extensive resection in patients with malignant astrocytomas, $1,2,7,8,16,17,20,22,24,25,36,37$ whereas other studies have failed to show this benefit. $5,6,13-15,23,30,34,40$ Still others have indicated that age and preoperative KPS score are better predictors of a favorable outcome than extent of resection. ${ }^{21,35}$ These results are complicated by that fact that the extent of resection is usually based on the surgeon's description rather than on postoperative imaging. ${ }^{17}$ Several in-depth reviews of the literature have cited a number of flaws in design and analysis in papers dealing with the extent of glioma resection..$^{21,30,34}$

Data regarding the potential of neuronavigation to improve the outcomes of glioma treatment are minimal. One retrospective study of 76 patients in which neuronavigation and extent of resection were considered indicated a survival advantage of both GTR (16 months compared with 9 months) and the use of neuronavigation (16 months compared with 10 months). ${ }^{24}$ Given the potential survival advantage of more extensive resection in patients with high-grade gliomas, we hypothesize that the use of intraoperative imaging technology or neuronavigation systems allows for more complete resections and, in turn, increased survival and improved outcome in these patients.

\section{Clinical Material and Methods}

\section{The Registry}

The GO Project is a multicenter, longitudinal, observational registry designed to track patterns of clinical practice and outcomes in patients who undergo surgery for malignant glioma. ${ }^{3}$ One hundred fifty-seven self-selected physicians at 57 clinical sites volunteered to enroll patients in the GO Project through a process approved by an institutional review board. ${ }^{27}$ The patient's informed consent was required for participation in the study.

Both physicians and patients completed standardized data sheets in the immediate postoperative period and then at 3-month intervals, for a total 24-month follow-up period. Data included demographic information, preoperative symptoms, functional status, treatment, medication status, postoperative complications, and patient satisfaction. Tumor-specific data included lesion size, location, histological grade, and pathological findings. Data regarding the surgical procedure, including type of procedure performed and the use of image guidance, were also recorded. Quality of life was measured using validated questionnaires, including the Short Form-36 Health Status Survey ${ }^{39}$ and the GO Questionnaire. ${ }^{3}$ Physicians at each site were educated by a coordinator to obtain standardized responses to the questions.

Enrollment criteria included adult patients undergoing biopsy sampling or a first or second resection for one of the following primary brain tumors: GBM, anaplastic astrocytoma, anaplastic oligodendroglioma, mixed anaplastic oligo/astrocytoma, or other anaplastic glioma. All patients were at least 18 years of age and were judged capable of giving informed consent. The GO Project began collecting patient data in 1997 and stopped enrolling patients in 2001. Our analysis included patients enrolled between December 1997 and July 2000.

The primary outcome measures included the following: 1) survival; 2) complications, including depression, seizures, and deep venous thrombosis; 3 ) quality of life over time; 4) patient satisfaction; 5) participation in clinical trials; 6 ) use of alternative therapies; and 7) duration of hospital stay. ${ }^{27}$

\section{Patient Selection}

Data obtained in 486 patients were analyzed in this study. Two groups of patients were compared: those with and those without IGR. For inclusion in this study, patients must have undergone a craniotomy for tumor resection or biopsy sampling. Patients who had undergone a stereotactic biopsy procedure performed in a bur-hole or twist-drill fashion were excluded. The type of image guidance used was not specified in the GO data. Outcome parameters included disposition of the patient at discharge; duration of hospital stay; neurological status; KPS 
score at 6,9, and 12 months postoperatively; and death at 6,9 , and 12 months postoperatively.

\section{Statistical Methods}

Categorical variables are expressed as frequencies and percentages, and continuous variables are expressed as a median. For univariate analysis, categorical variables were analyzed using the chi-square test or the Fisher exact test, and continuous variables were analyzed using a t-test in addition to the Wilcoxon rank-sum test. For multivariate analysis, variables were screened for entry into the logistic regression model by using the chi-square test. For the multiple linear regression model predicting duration of hospital stay, a log transformation of the dependent variable was used due to the distribution. The model was then assessed for goodness of fit by using regression statistical diagnostic methods, including the Cook-Weisberg test for heteroscedasticity. The statistical analysis was performed using commercially available statistical software (SAS version 8.2; SAS Institute, Cary, NC).

\section{Results}

Table 1 summarizes the clinical characteristics of all 486 patients, 305 of whom had standard resections without image guidance, whereas 172 had image-guided craniotomies. Parts of the data were missing for nine patients, which accounts for the variability in the numbers of patients included in the analysis. Patients who underwent IGR were significantly younger than those who did not (50 years compared with 56 years; $p<0.01$ ). They were also less likely to have certain clinical symptoms such as altered level of consciousness (12.2\% compared with $21.3 \% ; \mathrm{p}<0.02)$ or memory loss $(30.2 \%$ compared with $40.0 \% ; p<0.04)$. Patients who underwent IGR were more likely to present with seizures than those who underwent standard resections (39.5\% compared with $27.2 \%$; $\mathrm{p}<0.01)$. They were also more likely to have a tumor less than or equal to $4 \mathrm{~cm}$ in diameter $(51.7 \%$ compared with $36.4 \% ; \mathrm{p}<0.003$ ), and less likely to have a pathological finding of GBM $(65.7 \%$ compared with $79.7 \%$; $\mathrm{p}<$ 0.002 ).

The outcomes data are presented in Table 2. The median duration of hospital stay in patients who underwent IGR was 3 days, whereas in patients who did not undergo IGR it was 4 days ( $p<0.0001)$. Also, more patients who underwent IGR were discharged home with the ability to live independently than were those who were not treated with this procedure $(p=0.0003$, data unavailable for $18 \%$ of patients), and the death rate at 6,9 , and 12 months was significantly lower $(\mathrm{p}<0.05)$. In a subsequent multivariate analysis of the mortality rate, a diagnosis of pathological conditions other than GBM and younger age accounted for the decreases in this rate $(\mathrm{p}<0.0001)$. Multivariate analysis did confirm the roles of IGR $(p<0.008)$ and younger age $(p<0.003)$ as significant predictors of independent status on discharge home and decreased duration of hospital stay. Interestingly, a significantly higher percentage of patients who underwent IGR had subtotal resection than those in whom no image guidance was used (44\% compared with $35.1 \%$; $p<0.0001$, Fig 1).
TABLE 1

Clinical characteristics of 486 patients who underwent IGR or standard resection for high-grade glioma*

\begin{tabular}{llcc}
\hline \hline \multicolumn{1}{c}{ Characteristic } & IGR $(\%)$ & Non-IGR $(\%)$ & p Value $\dagger$ \\
\hline $\begin{array}{l}\text { median age (yrs) } \\
\text { altered level of }\end{array}$ & 50 & 56 & 0.0038 \\
$\quad$ consciousness & $21(12.2)$ & $65(21.3)$ & 0.0128 \\
headache & & & \\
memory loss & $93(54.1)$ & $180(59.0)$ & 0.3005 \\
language deficit & $52(30.2)$ & $122(40.0)$ & 0.0349 \\
seizures & $48(27.9)$ & $104(34.1)$ & 0.1678 \\
cognitive changes & $68(39.5)$ & $83(27.2)$ & 0.0054 \\
sensory symptoms & $48(27.9)$ & $112(36.7)$ & 0.0522 \\
papilledema & $29(16.9)$ & $35(11.5)$ & 0.0966 \\
visual disturbance & $11(6.4)$ & $15(4.9)$ & 0.4927 \\
tumor diameter $<4 \mathrm{~cm}$ & $35(20.3)$ & $73(23.9)$ & 0.3742 \\
GBM based on & $89(51.7)$ & $111(36.4)$ & 0.0022 \\
$\quad$ histopathological & $113(65.7)$ & $243(79.7)$ & 0.0012 \\
$\quad$ & & & \\
\hline findings & & & \\
\hline
\end{tabular}

* Data recorded in 486 patients enrolled in the GO Project were analyzed; 172 underwent IGR and 305 underwent standard resection (non-IGR). Missing data account for the nine patients who were not included in the analysis. $\dagger$ Significant at $<0.05$.

\section{Discussion}

These data show a surprising lack of IGR in patients with malignant glioma. Image guidance was used during the resection in only $172(35 \%)$ of 486 patients in this study. Our findings in this study cannot answer precisely why image-guided surgery is not routine. However, we did not take into account the depth of tumor from the cortical surface, which may provide one explanation. Surgeons may be less likely to take the time to set up and use image-guidance technology for tumors that are superficial and easily identifiable at the cortex. Alternatively, because the role of extent of resection in GBMs is controversial, some surgeons may believe that IGR provides little benefit to this group of patients. Large tumors can be more easily located without image guidance, and the brain shift that occurs can cause radiographically depicted tumor margins to vary (sometimes significantly) from real margins. Surgeons may think that the time required for setting up is not worth any potential benefits that might be realized.

Preoperative clinical characteristics differed between patients who underwent IGR and those who did not. These differences are probably important factors in the decision to use image-guided surgical techniques. The IGR procedure was more likely to be used in younger patients presenting with seizures and a normal level of consciousness who had smaller, less aggressive tumors. In several studies these characteristics have been identified as conferring a better prognosis. , $^{28,30}$

This selection bias supports the suggestion that IGR is used to maximize treatment in patients with a more favorable prognosis. Analysis of postoperative outcomes is consistent with this pattern, in that patients who underwent IGR had a shorter duration of hospital stay, were more likely to be discharged home with the ability to live independently, and were less likely to die. It is not possible to separate the effects of IGR from the selection bias toward patients with a better prognosis, given the design of this 
TABLE 2

Outcomes in 486 patients who underwent IGR or standard resection for high-grade glioma*

\begin{tabular}{lccr}
\hline \hline \multicolumn{1}{c}{ Outcome Parameter } & IGR $(\%)$ & Non-IGR $(\%)$ & p Value $\dagger$ \\
\hline $\begin{array}{l}\text { discharge disposition home/ } \\
\text { independent }\end{array}$ & $130 / 146(89.0)$ & $186 / 252(73.8)$ & 0.0003 \\
median LOS (days) & 3 & 4 & $<0.0001$ \\
neurological status & & & \\
$\quad$ worse & $9 / 168(5.4)$ & $33 / 302(10.9)$ & 0.1270 \\
unchanged & $70 / 168(41.7)$ & $117 / 302(38.7)$ & 0.1270 \\
better & $89 / 168(53.0)$ & $152 / 302(50.3)$ & 0.1270 \\
wound infection & $0 / 169$ & $3 / 294(1.0)$ & 0.5571 \\
$\quad$ periop) & & & \\
median KPS score & 90 & 90 & 0.5571 \\
6 mos & 90 & 90 & 1.0000 \\
9 mos & 90 & 80 & 0.0391 \\
12 mos & & & \\
deaths postop & $88 / 175(50.3)$ & $189 / 310(61.0)$ & 0.0224 \\
6 mos & $87 / 167(52.1)$ & $188 / 302(62.3)$ & 0.0325 \\
9 mos & $83 / 154(53.9)$ & $183 / 284(64.4)$ & 0.0310 \\
12 mos &
\end{tabular}

* Data recorded in 486 patients enrolled in the GO Project were used; missing data account for the discrepancies in the numbers of patients analyzed. Abbreviation: LOS = length of stay.

$\dagger$ Significant at $<0.05$.

study. It is possible that the survival benefits of IGR have not yet been realized in the subgroup of patients with poorer prognoses. Image guidance, when applied to older patients with larger tumors and more severe symptoms at diagnosis, may help to improve this poor prognosis. Further study in this area is warranted. Currently, surgeons may be contributing to a self-fulfilling prophecy; patients with favorable characteristics are treated more aggressively and do better, whereas those with less favorable char- acteristics are treated less aggressively and thus are more likely to have a poor outcome.

Strangely, in this study, patients who underwent IGR were less likely to attain a GTR. Considering the premise that image guidance would allow better definition of the borders of the tumor and subsequently a more precise resection, we find this result paradoxical. It is impossible to determine the factors involved in this observation based on our data. We believe that brain shift during frameless stereotaxy may cause an intraoperative impression that GTR was achieved, and that only with postoperative imaging is it revealed that the resection was subtotal.

Along with brain shift, the number and placement of fiducial markers can critically alter the accuracy of neuronavigational systems. With a small number of clustered fiducials, error can range as high as $9.5 \mathrm{~mm} .{ }^{38}$ Newer trends toward intraoperative MR imaging and ultrasonography combined with frameless navigational systems may help resolve this issue. This finding also leads us to suggest that surgeons who do use image-guided techniques may rely too heavily on the imaging in determining the extent of the resection, perhaps ignoring cues provided by the appearance and texture of the tissue. It is important for surgeons to remember that even the best navigational systems and imaging cannot replace the keen eye and clinical judgment of a well-trained neurosurgeon.

Unfortunately the retrospective questionnaire design of this study does not allow evaluation of exactly which IGR techniques were used. Presumably, real-time techniques such as intraoperative MR imaging and ultrasonography would be more likely to approach GTR than frameless stereotaxy. It is also likely that newer advances in functional MR imaging and labeling techniques will allow for less postoperative morbidity from damage to eloquent structures. Finally, the inherent weakness of the

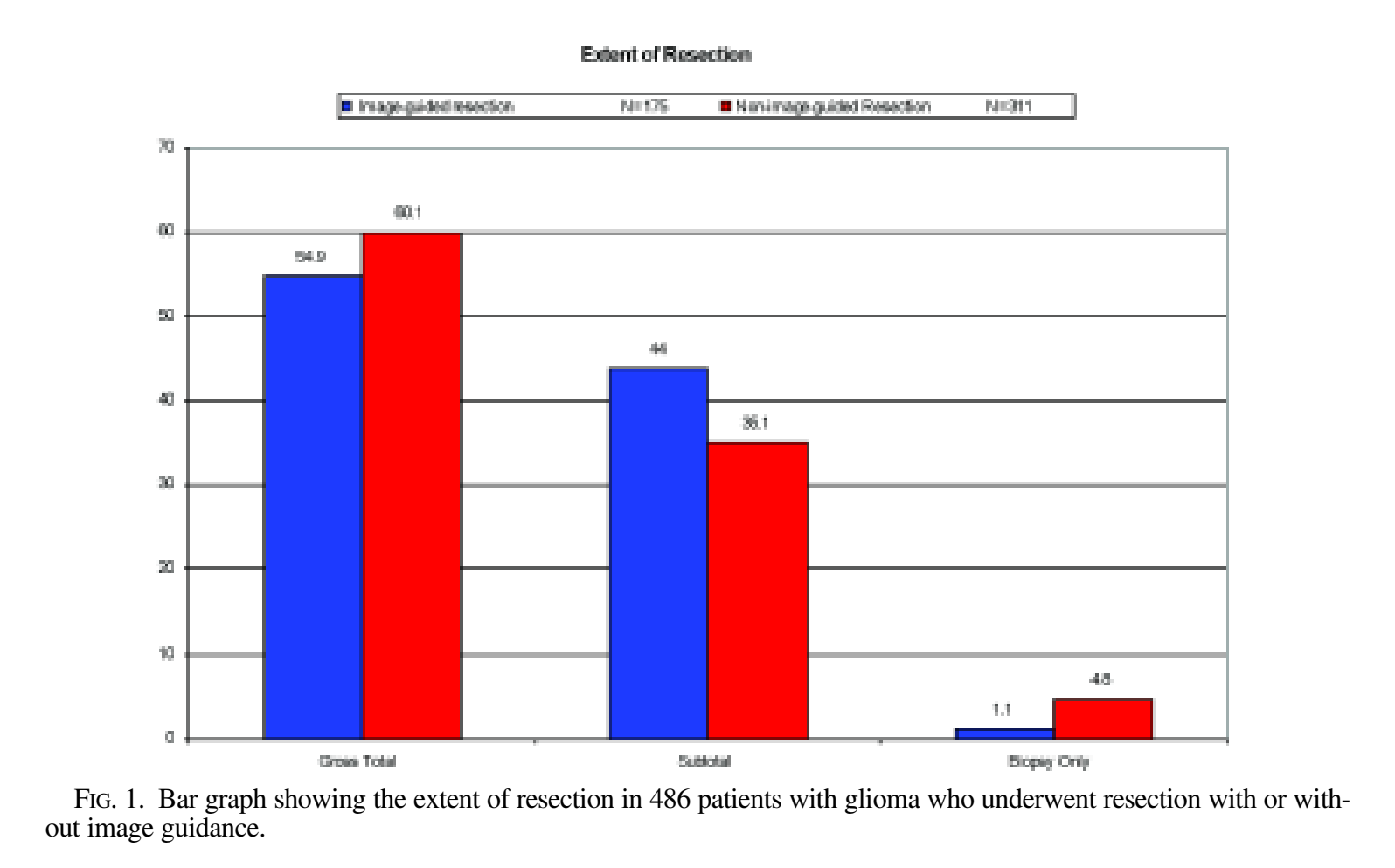


GO Project must be kept in mind: many diverse physicians enrolled only a few patients per doctor. As a result, considerable differences may exist between the IGR and non-IGR groups.

\section{Conclusions}

Patient characteristics associated with the use of IGR include younger age, seizures, and normal level of consciousness at presentation, tumor size less than $4 \mathrm{~cm}$ in diameter, and non-GBM findings on histopathological studies. Outcomes such as duration of hospital stay, discharge disposition, and mortality rates appear to be improved in patients who undergo IGR. These latter outcomes may be the result of patient selection rather than the IGR techniques themselves. We may not know the true benefits of navigational technology until it is applied to a wider range of patients, namely those with a worse prognosis. The fact that patients who underwent IGR in this study were more likely to have a suboptimal resection is an important reminder for surgeons not to rely too heavily on technology, and to use neuronavigation to augment clinical judgment. Neuronavigational technology may be more valuable if brain shift can be eliminated. As imaging technology improves and neuronavigational equipment becomes more precise, we will likely see an increased presence of these techniques in neurosurgical operating suites, and they will be used for a wider range of procedures.

\section{Acknowledgments}

We thank the GO Project Advisory Board for the use of the data and assistance with data analysis. The following are Project Advisors: Fred Anderson, Ph.D., University of Massachusetts Medical School; Anthony Asher, M.D., Carolina Neurosurgery \& Spine Associates; Mitchel Berger, M.D., University of California, San Francisco; Mark Bernstein, M.D., University of Toronto; Keith Black, M.D., Cedars Sinai Medical Center; Henry Brem, M.D., Johns Hopkins Medical Institutions; Faith Davis, Ph.D., University of Illinois at Chicago; Lawrence Hartman, M.D., Neurological Institute of Central Georgia; Fred Hochberg, M.D., Massachusetts General Hospital; Edward Laws Jr., M.D., University of Virginia; Kevin Lillehei, M.D., University of Colorado; N. Scott Litofsky, M.D., University of Missouri-Columbia; Jay Loeffler, M.D., Massachusetts General Hospital; Christina Meyers, Ph.D., University of Texas MD Anderson Cancer Center; Andrew Sloan, M.D., Wayne State University School of Medicine; and Michael Walker, M.D., National Institutes of Health.

\section{Disclosure}

The authors of this study have no financial stake in or affiliation with the manufacturer of any of the equipment mentioned within, nor do the authors advocate the use of any specific brand of equipment.

\section{References}

1. Albert FK, Forsting M, Sartor K, et al: Early postoperative magnetic resonance imaging after resection of malignant glioma: objective evaluation of residual tumor and its influence on regrowth and prognosis. Neurosurgery 34:45-61, 1994

2. Ammirati M, Vick N, Liao YL, et al: Effect of the extent of surgical resection on survival and quality of life in patients with supratentorial glioblastomas and anaplastic astrocytomas. Neurosurgery 21:201-206, 1987

3. Anderson FA Jr, The Glioma Outcomes Project Advisory Board: The Glioma Outcomes Project: a resource for measuring and improving glioma outcomes. Neurosurg Focus 4(6): E8, 1998

4. Black PM, Moriarty T, Alexander E III, et al: Development and implementation of intraoperative magnetic resonance imaging and its neurosurgical applications. Neurosurgery 41:831-845, 1997

5. Coffey RJ, Lunsford LD, Taylor FH: Survival after stereotactic biopsy of malignant gliomas. Neurosurgery 22:465-473, 1988

6. Curran WJ Jr, Scott CB, Horton J, et al: Recursive partitioning analysis of prognostic factors in three Radiation Therapy Oncology Group malignant glioma trials. J Natl Cancer Inst 85: 704-710, 1993

7. Daneyemez M, Gezen F, Canakci Z, et al: Radical surgery and reoperation in supratentorial malignant glial tumors. Minim Invasive Neurosurg 41:209-213, 1998

8. Devaux BC, O'Fallon JR, Kelly PJ: Resection, biopsy, and survival in malignant glial neoplasms. A retrospective study of clinical parameters, therapy, and outcome. J Neurosurg 78: 767-775, 1993

9. Ellison DW, Steart PV, Bateman AC, et al: Prognostic indicators in a range of astrocytic tumors: an immunohistochemical study with Ki-67 and p53 antibodies. J Neurol Neurosurg Psychiatry 59:413-419, 1995

10. Ganslandt $\mathrm{O}$, Behari S, Gralla J, et al: Neuronavigation: concept, techniques and applications. Neurol India 50:244-255, 2002

11. Golfinos JG, Fitzpatrick BC, Smith LR, et al: Clinical use of a frameless stereotactic arm: results of 325 cases. J Neurosurg 83:197-205, 1995

12. Haberland N, Ebmeier K, Hliscs R, et al: Neuronavigation in surgery of intracranial and spinal tumors. J Cancer Res Clin Oncol 126:529-541, 2000

13. Hess KR: Extent of resection as a prognostic variable in the treatment of gliomas. J Neurooncol 42:227-231, 1999

14. Hollerhage HG, Zumkeller M, Becker M, et al: Influence of type and extent of surgery on early results and survival time in glioblastoma multiforme. Acta Neurochir (Wien) 113:31-37, 1991

15. Huber A, Beran H, Becherer A, et al: [Supratentorial glioma: analysis of clinical and temporal parameters in 163 cases.] Neurochirurgia (Stuttg) 36:189-193, 1993 (Ger)

16. Jeremic B, Grujicic D, Antunovic V, et al: Influence of extent of surgery and tumor location on treatment outcome of patients with glioblastoma multiforme treated with combined modality approach. J Neurooncol 21:177-185, 1994

17. Keles GE, Anderson B, Berger MS: The effect of extent of resection on time to tumor progression and survival in patients with glioblastoma multiforme of the cerebral hemisphere. Surg Neurol 52:371-379, 1999

18. Keles GE, Berger MS: Advances in neurosurgical technique in the current management of brain tumors. Semin Oncol 31: 659-665, 2004

19. Keles GE, Lamborn KR, Berger MS: Low-grade hemispheric gliomas in adults: a critical review of extent of resection as a factor influencing outcome. J Neurosurg 95:735-745, 2001

20. Kiwit JC, Floeth FW, Bock WJ: Survival in malignant glioma: analysis of prognostic factors with special regard to cytoreductive surgery. Zentralbl Neurochir 57:76-88, 1996

21. Kowalczuk A, Macdonald RL, Amidei C, et al: Quantitative imaging study of extent of surgical resection and prognosis of malignant astrocytomas. Neurosurgery 41:1028-1038, 1997

22. Kreth FW, Berlis A, Spiropoulou V, et al: The role of tumor resection in the treatment of glioblastoma multiforme in adults. Cancer 86:2117-2123, 1999

23. Kreth FW, Warnke PC, Scheremet R, et al: Surgical resection 
and radiation therapy versus biopsy and radiation therapy in the treatment of glioblastoma multiforme. J Neurosurg 78: 762-766, 1993

24. Kurimoto M, Hayashi N, Kamiyama H, et al: Impact of neuronavigation and image-guided extensive resection for adult patients with supratentorial malignant astrocytomas: a singleinstitution retrospective study. Minim Invasive Neurosurg 47:278-283, 2004

25. Lacroix M, Abi-Said D, Fourney DR, et al: A multivariate analysis of 416 patients with glioblastoma multiforme: prognosis, extent of resection, and survival. J Neurosurg 95:190-198, 2001

26. Lindseth F, Lango T, Bang J, et al: Accuracy evaluation of a 3D ultrasound-based neuronavigation system. Comput Aided Surg 7:197-222, 2002

27. Litofsky NS, Farace E, Anderson F Jr, et al: Depression in patients with high-grade glioma: results of the Glioma Outcomes Project. Neurosurgery 54:358-367, 2004

28. Mitchell P, Ellison DW, Mendelow AD: Surgery for malignant gliomas: mechanistic reasoning and slippery statistics. Lancet Neurol 4:413-422, 2005

29. Nabavi A, Black PM, Gering DT, et al: Serial intraoperative magnetic resonance imaging of brain shift. Neurosurgery 48: 787-798, 2001

30. Nazzaro JM, Neuwelt EA: The role of surgery in the management of supratentorial intermediate and high-grade astrocytomas in adults. J Neurosurg 73:331-344, 1990

31. Nimsky C, Ganslandt O, Cerny S, et al: Quantification of, visualization of, and compensation for brain shift using intraoperative magnetic resonance imaging. Neurosurgery 47: 1070-1080, 2000

32. Nimsky C, Ganslandt O, Fahlbusch R: Functional neuronavigation and intraoperative MRI. Adv Tech Stand Neurosurg 29: 229-263, 2004

33. Nimsky C, Ganslandt $\mathrm{O}$, Kober $\mathrm{H}$, et al: Intraoperative magnet- ic resonance imaging combined with neuronavigation: a new concept. Neurosurgery 48:1082-1091, 2001

34. Quigley MR, Maroon JC: The relationship between survival and the extent of the resection in patients with supratentorial malignant gliomas. Neurosurgery 29:385-389, 1991

35. Salminen E, Nuutinen JM, Huhtala S: Multivariate analysis of prognostic factors in 106 patients with malignant glioma. Eur J Cancer 32A:1918-1923, 1996

36. Scott JN, Rewcastle NB, Brasher PM, et al: Which glioblastoma multiforme patient will become a long-term survivor? A population-based study. Ann Neurol 46:183-188, 1999

37. Shinoda J, Sakai N, Murase S, et al: Selection of eligible patients with supratentorial glioblastoma multiforme for gross total resection. J Neurooncol 52:161-171, 2001

38. Steinmeier R, Rachinger J, Kaus M, et al: Factors influencing the application accuracy of neuronavigation systems. Stereotact Funct Neurosurg 75:188-202, 2000

39. Ware JE Jr, Sherbourne CD: The MOS 36-item short-form health survey (SF-36). I. Conceptual framework and item selection. Med Care 30:473-483, 1992

40. Weir B: The relative significance of factors affecting postoperative survival in astrocytomas, grades 3 and 4 . J Neurosurg 38: 448-452, 1973

Manuscript received January 17, 2006.

Accepted in final form March 16, 2006.

The GO Project is supported by unrestricted educational grants from Aventis Pharmaceuticals, Inc., Bridgewater, New Jersey, and Guilford Pharmaceuticals, Inc., Baltimore, Maryland.

Address reprint requests to: N. Scott Litofsky, M.D., Division of Neurological Surgery, University of Missouri School of Medicine, One Hospital Drive, N521, Columbia, Missouri 65212. email: litofskyn@health.missouri.edu. 